\title{
Mot du directeur de la rédaction
}

\section{P.-H. Benamou}

(C) Springer-Verlag 2009

Chers collègues,

La revue «Médecine et chirurgie du pied - Foot Medicine and Surgery (SFMCP), devient une revue internationale avec deux nouvelles indexations :

- Science Citation Index Expanded, connu également sous le nom SciSearch ${ }^{\circledR}$, hébergé sur le site Internet « ISI Web of Knowledge » qui permet de connaître le nombre de citations d'un article de la revue (http://apps.isiknowledge.com/) ;

- Journal Citation Reports-Science Edition (JCR) qui établit le fameux impact factor pour les revues.

Ces indexations, outre Embase (Excerpta Medica), Pascal (INIST-CNRS), Scopus, GoogleScholar, constituent des arguments supplémentaires de poids pour l'indexation dans Medline.
La SFMCP a su se montrer solidaire de l'AFCP (Association française de chirurgie du pied), lors des dernières élections de l'EFAS (European Foot and Ankle Society), en octobre 2008 à Villamoura, et je souhaiterais que cette revue soit celle de tous les médecins et de tous les chirurgiens du pied.

Je vous demande également de contribuer le plus activement possible à faire connaître les articles parus dans la revue «Médecine et chirurgie du pied», afin d'augmenter leurs citations dans les bibliographies et, ainsi, de permettre à la revue de démarrer avec un impact factor le plus élevé possible.

Ce n'est pas chose facile pour l'équipe de rédaction de maintenir une revue à un tel niveau ; aussi, je vous demande à tous de la considérer comme la vôtre, indépendamment de toute polémique stérile. 